\title{
Computational algorithm for hybrid rocket motor preliminary design
}

\author{
Mohamed Sakr \\ School of Built Environment, Ulster University, Jordanstown Campus, \\ BT370QB, UK \\ sakr-m@ulster.ac.uk
}

\begin{abstract}
Hybrid rockets present a compromise between solid and liquid rockets. It has a simpler design than liquid rockets and higher performance than solid rockets. It is constructed from an oxidizer tank followed by a solid fuel grain. Therefore, the combustion is controlled.

This paper presents an algorithm to construct the design space of the hybrid rocket motor for a single cylindrical port. A simple regression rate formula is used to treat the combustion. The inner diameter of the grain, outer diameter of the grain, and length of the grain are used as parameters to construct the design space and a single design point is chosen. The performance of this design point is presented with the combustion time.
\end{abstract}

Keywords: hybrid rocket motor, regression rate, nitrous oxide, paraffin wax

\section{Introduction}

There are three main concepts for designing rocket motors: solid motor, liquid motors, and hybrid motors. In the solid motors, both the oxidizer and the fuel are combined in the form of solid grain. This design is simple, however there is risk of explosion in the manufacturing process. The performance of the solid motor, in terms of the specific impulse, is low. Once the motor is ignited, the combustion is not controllable. In the liquid motors, both the fuel and the oxidizer are separated in the liquid form. This design is safe, but it is complicated. Its performance, in terms of the specific impulse, is high and there is high controllability at the combustion. In the hybrid motor, the fuel is stored in a solid phase, while the oxidizer is stored either in a gas or in a liquid phase. Hybrid motors present a compromise between the solid and the liquid motors. The design is simpler than the liquid motor and it is safe with moderate performance, in terms of the specific impulse, and there is controllability at the combustion.

Hybrid rockets could be used in different applications like space propulsion, as in the spaceship one and two. A Schematic of the hybrid rocket 
motor is shown in Fig. 1. It consists of an oxidizer tank followed by the fuel grain and the rocket nozzle. The grain presents the combustion chamber, where the reaction occurs due to diffusion in the flow boundary layer. The oxidizer is injected through an injection nozzle, where the oxidizer mass flow rate $\left(\dot{m}_{o x}\right)$ is determined based on the injection nozzle geometry and the difference between the pressure of the tank and the pressure of the combustion chamber. The grain could be designed with a single port or multiport. Different shapes could be used for designing the grain like cylinder or star or others. The shape of the design would change the combustion power due to changing the contact area between the fuel and the oxidizer. The fuel mass flow rate is determined based on the regression rate $(\dot{r})$, which represents the burning rate of the grain. It is parameterized with the oxidizer flux $\left(G_{o x}\right)$ using experiments.

In the present paper an algorithm to construct the design space of the hybrid rocket motor is presented. The combustion is treated using a simple form of the regression rate. The flow in the nozzle is presented using the isentropic relations.

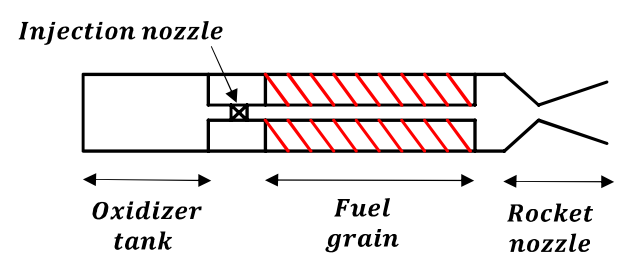

Fig. 1. Schematic of the hybrid rocket motor.

\section{Design algorithm}

The objective of this section is to determine the design space of the hybrid rocket motor. The oxidizer and fuel types, the pressure of the tank, the pressure of the combustion chamber, and the grain shape are chosen. In the current study, the oxidizer is chosen as nitrous oxide and the fuel is chosen as paraffin wax. The pressure of the tank $\left(p_{t a}\right)$ is chosen as 50 bar, while the pressure of the combustion chamber $\left(p_{c}\right)$ is chosen as 38 bar [1]. The grain is chosen as a single cylindrical port. The algorithm is performed sequentially starting with calculating the oxidizer mass flow rate from the tank through the injection nozzle, as shown in Eq. (1) [2].

$$
\dot{m}_{o x}=c d A_{i n j} \sqrt{2 \rho_{l}\left(p_{t a}-p_{c}\right)}
$$

$c d$ is the discharge coefficient of the injection nozzle, $A_{i n j}$ is the cross-section area of the injection nozzle, and $\rho_{l}$ is the density of the liquefied nitrous oxide. The oxidizer flux is calculated in Eq. (2) as the mass flow rate per the wetted area of the grain. 


$$
G_{o x}=\frac{\dot{m}_{o x}}{\pi D_{i n} L_{p}}
$$

$D_{i n}$ is the inner diameter of the grain and $L_{p}$ is the length of the grain. The regression rate is calculated from Eq. (3) [3].

$$
\dot{r}=0.104 G_{o x}^{0.67}
$$

The fuel mass flow rate $\left(\dot{m}_{f}\right)$ is calculated from Eq. (4).

$$
\dot{m}_{f}=\rho_{f} \pi D_{\text {in }} L_{p} \dot{r}
$$

$\rho_{f}$ is the fuel density. The total mass flow rate $(\dot{m})$ is the sum of the oxidizer and fuel mass flow rates. Assuming the rocket nozzle starts with the outer diameter of the rocket, then the Mach number at the beginning of the nozzle $\left(M_{1}\right)$ is calculated from Eq. (5).

$$
M_{1}=\frac{4 \dot{m}}{\pi \rho_{c} D_{R}^{2} \sqrt{\gamma_{c} R_{c} T_{c}}}
$$

$\rho_{c}$ is the density of the combustion product, $D_{R}$ is the outer diameter of the rocket, $\gamma_{c}$ is the specific heat of the combustion product, $R_{c}$ is the gas constant of the combustion product, and $T_{c}$ is the temperature of the combustion product. Using $M_{1}$, the total thermodynamic state could be determined as shown in Eqs. (6)-(8) [4].

$$
p_{t}=p_{c}\left[1+\frac{\gamma_{c}-1}{2} M_{1}^{2}\right]^{\frac{\gamma_{c}}{\gamma_{c}-1}}
$$

$$
T_{t}=T_{c}\left[1+\frac{\gamma_{c}-1}{2} M_{1}^{2}\right]
$$

$$
\rho_{t}=\rho_{c}\left[1+\frac{\gamma_{c}-1}{2} M_{1}^{2}\right]^{\frac{1}{\gamma_{c}-1}}
$$

$p_{t}, T_{t}$, and $\rho_{t}$ are the total pressure, total temperature, and total density. Assuming the rocket nozzle exit pressure $\left(p_{e}\right)$ is the ambient pressure, then the Mach number at the nozzle exit $\left(M_{2}\right)$ is calculated from Eq. (9).

$$
M_{2}=\sqrt{\left(\left(\frac{p_{e}}{p_{t}}\right)^{\frac{1-\gamma_{c}}{\gamma_{c}}}-1\right) \frac{2}{\gamma_{c}-1}}
$$

The temperature at the nozzle exit $\left(T_{2}\right)$ is calculated from Eq. (10) [4].

$$
T_{2}=\frac{T_{t}}{1+\frac{\gamma_{c}-1}{2} M_{2}^{2}}
$$

The density at the nozzle exit $\left(\rho_{2}\right)$ is calculated from Eq. (11) [4].

$$
\rho_{2}=\frac{\rho_{t}}{\left[1+\frac{\gamma_{c}-1}{2} M_{2}^{2}\right]^{\frac{1}{\gamma_{c}-1}}}
$$

The throttle area $\left(A^{*}\right)$ of the nozzle is calculated from Eq. (12) [4]. 


$$
\begin{aligned}
& A^{*} \\
& =\frac{\pi}{4} D_{R}^{2}\left[\frac{\gamma_{c}+1}{2}\right]^{\frac{\gamma_{c}+1}{2 \gamma_{c}-2}}[1 \\
& \left.+\frac{\gamma_{c}-1}{2} M_{1}^{2}\right]^{\frac{-\gamma_{c}-1}{2 \gamma_{c}-2}} M_{1}
\end{aligned}
$$

The area of the nozzle exit $\left(A_{2}\right)$ is calculated from Eq. (13) [4].

$$
\begin{aligned}
& A_{2} \\
& =\frac{A^{*}}{M_{2}}\left[\frac{\gamma_{c}+1}{2}\right]^{\frac{-\gamma_{c}-1}{2 \gamma_{c}-2}}[1 \\
& \left.+\frac{\gamma_{c}-1}{2} M_{2}^{2}\right]^{\frac{\gamma_{c}+1}{2 \gamma_{c}-2}}
\end{aligned}
$$

The velocity at the nozzle exit $\left(V_{2}\right)$ is calculated from Eq. (14).

$$
V_{2}=\frac{\dot{m}}{\rho_{2} A_{2}}
$$

The thrust of the rocket $(T)$ is calculated from Eq. (15).

$$
T=\dot{m} V_{2}
$$

The above equations represent a system that could be solved to build the design space of the hybrid rocket.

\section{Testing of the algorithm}

Solving the above equations with the inner diameter of the grain, the outer diameter of the grain, and the length of the grain as input parameters, the rocket design space is determined. The rocket thrust is given in Fig. 2 against different initial inner diameter of the grain, where the length of the grain is taken as a fixed value of $10 \mathrm{~m}$ and the outer diameter of the grain is taken as a fixed value of 4.5 $m$.

The performance of the rocket with the change in the inner diameter of the grain during burning is shown in Fig. 3, under the assumption of a constant pressure of the tank. The initial inner diameter of the grain is taken as $2 m$, and the burning time is taken as $180 \mathrm{~S}$. During this burning time, the regression rate is changed due to the change in the inner diameter. 


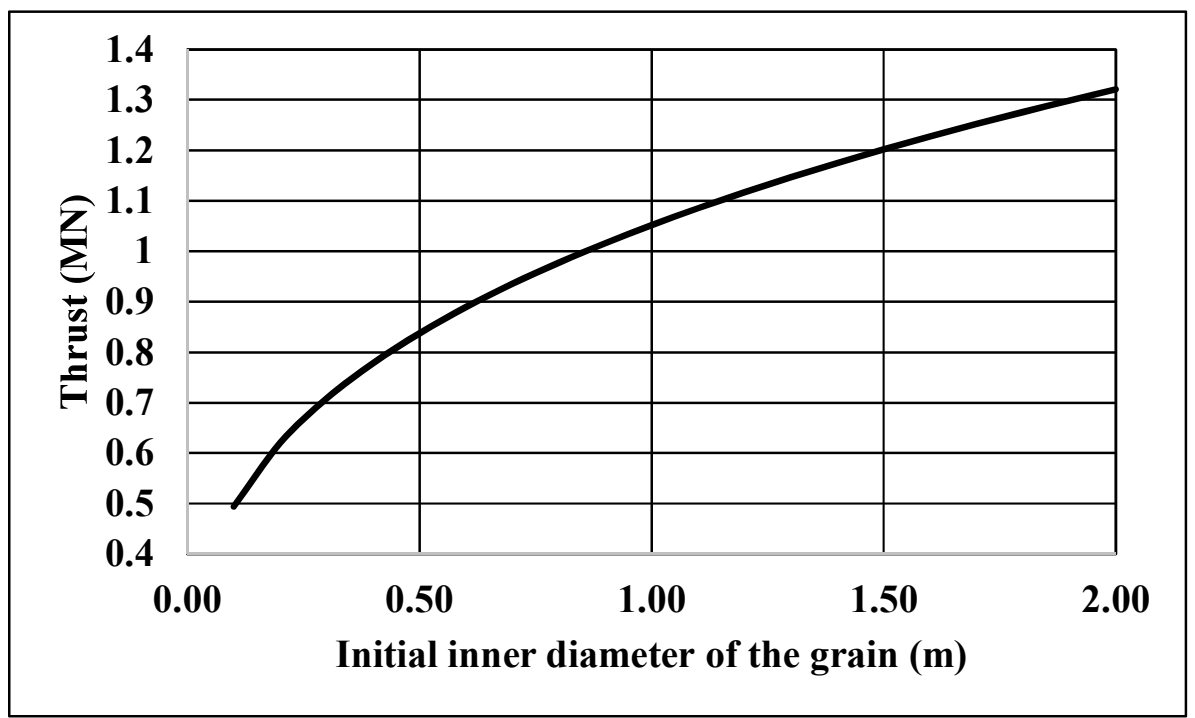

Fig. 2. Rocket thrust for different initial inner diameter of the grain.

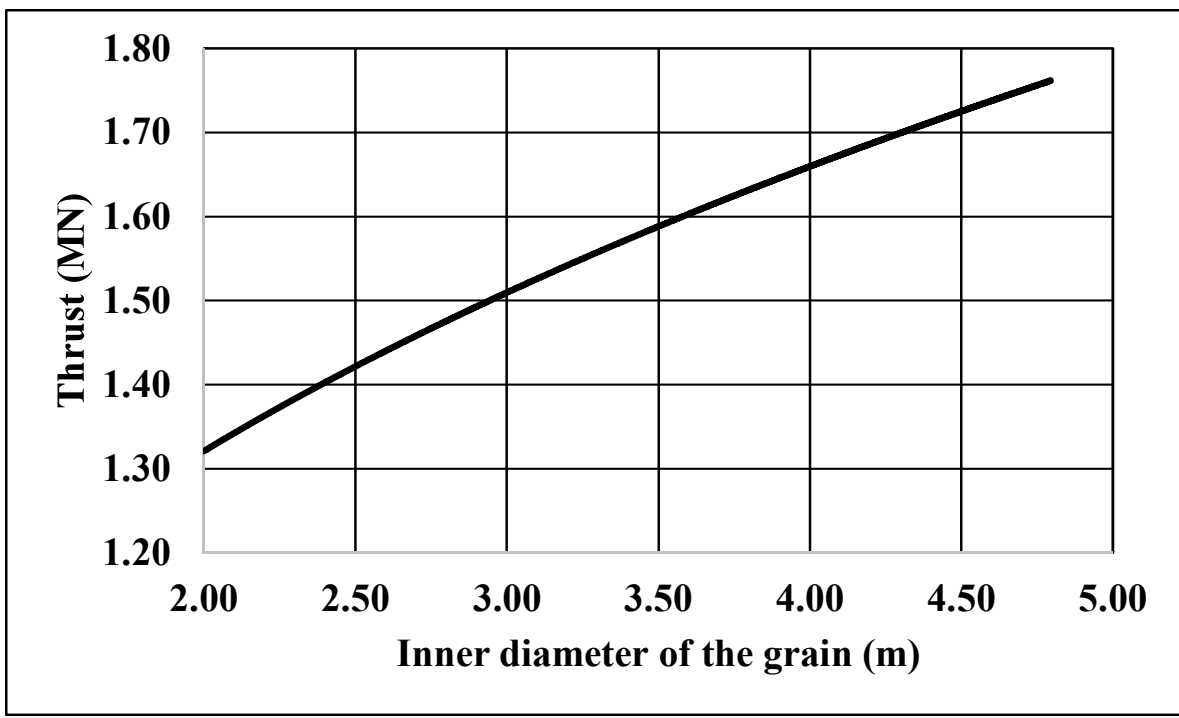

Fig. 3. Rocket thrust with the change in the inner diameter of the grain during burning.

\section{Conclusions}

A computational algorithm to calculate the design space of the hybrid rocket motor is presented. The nitrous oxide is chosen as the oxidizer and the paraffin wax is chosen as the fuel. The shape of the grain is chosen as a single cylindrical port. A simple regression rate formula is used to model the combustion and the isentropic relations are used to model the flow in the nozzle. The design space is constructed in terms of the inner diameter of the grain, the outer diameter of the grain, and the length of the grain. The rocket thrust is determined against the initial inner diameters of the grain for fixed values of the outer diameter of the grain and the length of the grain. The performance of the rocket is also 
determined with the change in the inner

diameter of the grain due to burning.

\section{Reference}

[1] R. Erickson, "Numerical modeling of a hybrid rocket," 2005.

[2] M. Gieras and A. Gorgeri, "Numerical modelling of the hybrid rocket engine performance," Propuls. Power Res., vol. 10, no. 1, pp. 15-22,
2021.

[3] S. Sisi and A. Gany, "Parametric investigation of a hybrid motor using paraffin and nitrous oxide," 54th Isr. Annu. Conf. Aerosp. Sci. 2014, vol. 2, no. January 2014, pp. 944-959, 2014.

[4] NASA, "Isentroic flow," Glenn Res. Cent., no. may, 2021. 